\title{
KörperLeibliche Dimensionen der Konstituierung von Grenzen
}

\author{
Anna Bea Burghard • Veronika Magyar-Haas · Rebecca Mörgen
}

Zusammenfassung: Im Kontext sozialpädagogischer Forschung und Theoriebildung bildet die theoriesystematische Reflexion der Verhältnisse von Körper/Leib und Grenzen eine Leerstelle. Diese Zusammenhänge werden im Beitrag vor dem Hintergrund anthropologischer, poststrukturalistischer, sozialontologischer sowie praxeologischer Theorieperspektiven beleuchtet. In diesen Bestimmungsversuchen erweist sich der Körper zum einen als Grenze sowie in seiner Grenzhaftigkeit, zum anderen sind Körper an der Konstitution von Grenzen ebenfalls beteiligt. Es wird aufgezeigt, dass und wie mittels Markierungen bestimmter Aspekte von und an Körpern symbolische Grenzen, während mit Körpern - wie etwa bei der Konstituierung von Kollektivität und Gemeinschaft - zugleich soziale Grenzen konstruiert werden. Im Zuge der Analysen werden insbesondere Fragen nach der hegemonialen Wahrnehmbarkeit, der (Un)Sichtbarkeiten und des (Un)Sagbaren diskutiert. Die theoriegeleiteten Überlegungen tragen dazu bei, Soziale Arbeit als ,grenzbearbeitende Wissenschaft` körpertheoretisch zu reflektieren.

Schlüsselwörter: Körper/Leib · Grenze · Verletzbarkeit · Wahrnehmbarkeit/(Un)Sichtbarkeit · Normen · Macht

\section{Bodily dimensions of the constitution of boundaries}

Abstract: Reflection on the relations between body and boundaries in terms of the systematics of theory constitutes a lacuna in the context of socio-pedagogical research and theorizing. In this article, these interrelationships will be examined in the light of anthropological, post-structuralist,

(C) Die Autor(en) 2014. Dieser Artikel ist auf Springerlink.com mit Open Access verfügbar.

V. Magyar-Haas $(\bowtie)$

Institut für Erziehungswissenschaft, Lehrstuhl für Pädagogik-Sozialpädagogik, Universität Zürich, Freiestr. 36, 8032 Zürich, Schweiz

E-Mail: vmagyar@ife.uzh.ch

A. B. Burghard

Institut für Erziehungswissenschaft, Abt. II., Arbeitsbereich Sozialpädagogik,

Westfälische Wilhelms-Universität Münster,

Georgskommende 33, 48143 Münster, Deutschland

E-Mail: annabea.burghard@uni-muenster.de

R. Mörgen

Institut für Erziehungswissenschaft, Lehrstuhl für Außerschulische Bildung und Erziehung,

Universität Zürich,

Freiestr. 36, 8032 Zürich, Schweiz

E-Mail: rmoergen@ife.uzh.ch 
socio-ontological and praxeological theoretical perspectives. In these attempts at definition, the body on the one hand reveals itself as a boundary, or is perceived in its exposure; on the other hand bodies are also involved in the constitution of boundaries. The paper shows how symbolic boundaries are constructed by the highlighting of particular aspects of and on bodies, while at the same time social boundaries are constructed with bodies-for example in the process of constituting collectivity and community. The analyses will focus on questions of hegemonic perceptibility, (in)visibility and the (un)speakable/(un)sayable. These theory-driven considerations will contribute to reflect on social work as a 'boundary-work science' in terms of theories of the body.

Keywords: Body (that I have/that I am) $\cdot$ Boundary $\cdot$ Vulnerability $\cdot$

Perceptibility/(In)Visibility · Norms · Power

Grenzziehungen und Grenzauflösungen, so lässt sich politik-, geschichts-, und sozialwissenschaftlichen Argumentationen entnehmen, sind Produkte und Produzenten von Auseinandersetzungen und Zuschreibungen und stellen so Prozesse der Machtdemonstration und -ausübung dar. Entsprechend lassen sich Grenzen als „Ausdruck[...] politischer Macht- und Herrschaftsverhältnisse“ (Kessl 2009, S. 47) bestimmen. Die Aufgabe, ,permanent das Gefüge der Macht zu dechiffrieren und zu problematisieren, um die Grenzen des Bestehenden zu erweitern, zu vervielfältigen oder auch zu unterwandern“" schreibt etwa Kessl (2009, S. 48) der Disziplin und Profession Sozialer Arbeit zu. Entsprechend dieser Postulierung lässt sich Soziale Arbeit als ,grenzbearbeitende Wissenschaft` begründen (vgl. Kessl und Maurer 2010; Heite 2013; Magyar-Haas 2012). Neben dem Aspekt der Macht werden Grenzen auch hinsichtlich ihrer Materialität, Räumlichkeit, Territorialität sowie in Bezug auf soziale Ungleichheit thematisiert. Unberücksichtigt bleibt dabei die Bedeutung des KörperLeibes ${ }^{1}$ bei der Konstituierung von Grenzen.

Da eine systematische Analyse des Verhältnisses von Grenze und KörperLeib in der sozialpädagogischen Forschung und Theoriebildung eine Leerstelle markiert, fokussiert der vorliegende Beitrag diese Zusammenhänge ${ }^{2}$ mit dem Anspruch, einige Systematisierungsvorschläge zu bieten. Diese Annäherung wird unter zwei Fragestellungen vorgenommen: Inwiefern lässt sich KörperLeib selbst als Grenze bestimmen (Kap. 1)? Welche Bedeutung kommt KörperLeibern bei der Frage nach der Hervorbringung von Grenzen ${ }^{3}$ zu (Kap. 2 und 3)?

Zunächst werden verschiedene Bedeutungsfacetten des Körpers aus divergenten theoretischen Ansätzen systematisch beleuchtet, um die Relevanz des Körpers als sozialwissenschaftlichen - und so auch sozialpädagogischen - Forschungsgegenstand hinsichtlich der Konstituierung von Grenzen zu perspektivieren (1). Vor diesem Hintergrund eröffnet

1 Der Begriff des KörperLeibes wird hier verwendet, um auf die Verschränkung von Körper und Leib, zwischen denen in phänomenologischen und anthropologischen Auseinandersetzungen analytisch differenziert wird, hinzuweisen (vgl. Jäger 2004).

2 Auf der Jahrestagung der Kommission Sozialpädagogik 2013 in Tübingen wurden von den Autorinnen drei separate Vorträge zur Diskussion gestellt. Für diese Zeitschrift wurde auf dieser Basis und unter Berücksichtigung der kritischen Anregungen bei den Podiumsdebatten ein Beitrag zur Thematik des Aufsatzes gemeinsam verfasst.

3 Entsprechend wird nicht davon ausgegangen, dass Grenzen etwas ,Präexistentes` seien, sondern dass sie hervorgebracht werden. 
sich die Frage, inwiefern Markierungen an und über Körper (2) sowie Praktiken mit Körpern (3) Grenzen hervorbringen. Abschließend folgt ein ausblickendes Resümee auf die in dem Beitrag rekonstruierten Dimensionen der Grenzkonstituierung über, an und mit Körper(n), um verschiedene grenzanalytische Perspektiven (vgl. Heite et al. 2013; Kessl und Maurer 2010) körpertheoretisch weiterzuführen (4).

\section{Be-Deutungsfacetten des Körpers vor dem Hintergrund von Grenzen}

KörperLeiber erlangen in analytischer Hinsicht in vier Theorieströmungen eine explizite Bedeutung: in anthropologischen, poststrukturalistischen, sozialontologischen sowie praxeologischen Zugängen ${ }^{4}$. Das Anliegen des vorliegenden Beitrages ist, diese Ansätze daraufhin zu befragen, wie in ihnen KörperLeiber gedeutet bzw. welche Bedeutungen diesen zugeschrieben werden. Zugleich wird es um die Frage gehen, wie in den relevant gesetzten Auslegungen das Verhältnis von KörperLeibern und Grenzen konzeptionalisiert wurde oder werden könnte. Die hier zu thematisierenden Perspektiven markieren verschiedene Konjunkturen der theoretischen Auslegungen von Körpern im Laufe des 20. Jahrhunderts.

\subsection{Körper als Grenze: Anthropologische Bestimmungen}

Im Folgenden wird Helmuth Plessners Bestimmung des Leibes als Grenze dargelegt sowie auf die Relevanz von Grenzsituationen, in denen der (verselbstständigte) KörperLeib agiere, eingegangen. Begründen lässt sich die Hervorhebung Plessners philosophischer Anthropologie zum einen damit, dass der Kategorie der Grenze in seinem als Hauptwerk angesehenen ,Die Stufen des Organischen und der Mensch“ von 1928 eine zentrale Bedeutung zukommt, zum anderen, dass Plessners Augenmerk auf soziale Situationen des Lachens und Weinens liegt, die als Grenzsituationen aufgefasst werden können.

In Bezugnahme auf den Begriff der Grenze, in der Frage nach dem „Verhältnis des begrenzten Körpers zu seiner Grenze“ (Plessner 1928/2003, S. 154), entfaltet Plessner die Differenz zwischen ,lebendigen“ und ,unlebendigen Dingen' und nimmt dabei folgende Definition vor: „Körperliche Dinge der Anschauung, an welchen eine prinzipiell divergente Außen-Innenbeziehung als zu ihrem Sein gehörig gegenständlich auftritt, heißen lebendig“ (Plessner 1928/2003, S. 138, herv. i. O.). Diese Auslegung wird in mehreren Schritten entfaltet. Zunächst setzt Plessner bei der „Gegenständlichkeit des fernen Gegenstandes, wie er der Wahrnehmung gegenüber gegeben ist“" (Fischer 2000, S. 271) an, also nicht bei dem Subjekt. Die ,,anschaulichen Grenzen“ von anorganischen, ,dinghaften“ Körpern würden da liegen, ,wo sie anfangen oder zu Ende sind. Die Grenze des Dinges ist sein Rand“ (Plessner 1928/2003, S. 151). ,Lebendige Körper“ wiederum hätten die Grenze, die Innen (gefasst als ,substanzieller, innerer Kern') und Außen (im Sinne äußerlich erscheinender Eigenschaften) scheidet, selbst als Eigenschaft (vgl. Plessner 1928/2003, S. 128 ff.). Also hört ein ,lebendiges Ding“ nicht an der Grenze auf, vielmehr

4 Diese Auswahl liegt primär in den Forschungsschwerpunkten der Verfasserinnen begründet. Auf weitere Perspektiven wird in den jeweiligen Abschnitten kontrastierend oder ergänzend hingewiesen. 
lässt sich die Grenze als „realisierte Beziehung zwischen Innen und Außen“ (Fischer 2000, S. 272), also in ihrer Verhältnishaftigkeit bestimmen. So subsumiert Plessner (1928/2003, S. 181): „Die Grenze gehört dem Körper selbst an, der Körper ist die Grenze seiner selbst und des Anderen und insofern sowohl ihm als dem Anderen entgegen". 5 Entsprechend werde das Lebewesen durch die Grenze nicht nur ,eingeschlossen ', sondern Anderen und Anderem gegenüber aufgeschlossen, mit ihnen in Verhältnis gesetzt; so ermögliche die Grenze gegenüber dem Anderen ein Öffnen und Schließen zugleich (vgl. Plessner 1928/2003, S. 181 ff). Für diese Möglichkeit, in die (physischen, ,hauthaften`) Grenzen gesetzt und zugleich über diese Grenzen hinaus und diesen Grenzen entgegengesetzt zu sein, prägte Plessner (1928/2003, S. 181 ff.) den Begriff der „Positionalität“ als Kennzeichen der Lebendigkeit. Während das „Leben des Tieres“ als „,zentrisch“ positioniert gefasst wird, sei „das Leben des Menschen, ohne die Zentrierung durchbrechen zu können, zugleich aus ihr heraus, exzentrisch. Exzentrizität ist die für den Menschen charakteristische Form seiner frontalen Gestelltheit gegen das Umfeld“ (Plessner 1928/2003, S. 364, herv. i. O.). Mit dieser Aussage wird auch das Verhältnis des Menschen zu seinem Körper als ein Zweifaches beschrieben: Er ist sein Körper im Sinne von Leib-Sein und hat seinen Körper im Sinne von Körper-Haben. So lebe der Mensch in einem „unaufhebbare[n] Doppelaspekt“ (Plessner 1928/2003, S. 365), als Körper und als Leib. Er sei stets mit seinem Körper an das Hier-und-Jetzt gebunden, „mit seinem Leib in den Umweltbezug eingebunden“ (Jäger 2004, S. 123) - und zugleich über dieses Verhältnis hinaus. Dabei sei der Leib ${ }^{6}$,als Ausdrucksfläche [...] auch bei den Tieren keine passive Hülle und Außenschicht, in die sich die Erregungen von innen hineinbeulen, sondern eine erlebte Grenzfläche gegen die Umwelt" (Plessner 1941/2003, S. 249). In dieser Perspektive wird der Leib als Austragungsort sozialer Verhältnisse erkennbar, als Eindruck und Ausdruck. ${ }^{7}$

Dass ,der Mensch` zu seinem Leib kein eindeutiges, sondern ein doppeldeutiges Verhältnis (zwischen sich und sich, zwischen ,Körpersein und Körperhaben') habe, plausibilisiert Plessner in seiner Studie über Lachen und Weinen (vgl. Plessner 1941/2003, S. 235 ff.). Auf diese expressiven Formen wird hier eingegangen, da sie von Plessner als „Grenzreaktionen“ (Plessner 1941/2003, S. 366) bezeichnet werden. Sie seien in unbeantwortbaren, aber nicht bedrohenden Situationen beobachtbar (vgl. Plessner 1941/2003, S. 276). In solchen ,Grenzlagen“ kapituliere der Mensch nicht als Person, aber als „LeibSeele-Einheit" und der Körper ,emanzipiere" sich von der Person, er übernehme die Antwort auf eine Situation, auf die er in anderer Weise nicht zu reagieren vermag (Plessner 1941/2003, S. 276). ,Der Mensch“ überlasse sich den beiden Grenzformen: „Er verfällt ins Lachen, er lässt sich fallen - ins Weinen“ (Plessner 1941/2003, S. 273, herv. i. O.) ${ }^{8}$.

5 Bereits diese Formulierungen vermögen auf ausgesetzte, grenzhafte Körper verweisen, die in aktuellen sozialontologischen Auseinandersetzungen prominent gesetzt werden (vgl. dazu 1.3).

6 Zur Weiterführung Plessners Differenzierung in phänomenologischen Debatten vgl. MagyarHaas 2013, S. $140 \mathrm{ff}$.

7 Weiterführend zur Konzeptionalisierung von Körper als Grenze bei Plessner vgl. Magyar-Haas 2012, S. 6 ff.

8 In der Analyse verschiedener Anlässe des Lachens und Weinens bestimmt Plessner Lachen als eine „Reaktion auf Unterbindung des Verhaltens durch unausgleichbare Mehrsinnigkeit der Anknüpfungsmöglichkeiten“, während er Weinen als Reaktion auf die „Unterbindung des 
In diesen Situationen sind Personen an ihre Grenzen gekommen, an die Grenze des sinnhaft Beantwortbaren, wo zu diesen Situationen zu viele oder gar keine Bezüge hergestellt werden können, in denen ein Abstand gerade noch eingenommen werden kann, doch die Antwort nicht mit dem Körper sondern des Körpers erfolgt (vgl. Plessner 1941/2003, S. 276). ${ }^{9}$

Wird in dieser anthropologischen Betrachtung Körper als Grenze, als (materielles) ,Produkt‘ eines Außen-Innen-Verhältnisses diskutiert, rücken im folgenden Abschnitt konträr dazu - poststrukturalistische Betrachtungsweisen auf den Körper in den Blick, die Körper nicht als ,natürliches Ding', sondern als ,Diskurseffekt', als Verschränkung von Macht und Wissen bestimmen. Dabei stellt sich auch die Frage nach der Materialisierung von Diskursen im und am Körper.

\subsection{Materialisierung von Diskursen im Körper: Poststrukturalistische Deutungen}

Inwiefern sich Körper als Produkt machtvoller Handlungen, welche sich „um den Körper, am Körper, im Körper" produzieren, begreifen lässt, legt Michel Foucault (1976, S. 41) in seinen Überlegungen zur Disziplinargesellschaft dar. Mit seiner genealogischen Betrachtung und Analyse der „Mikrophysik der Macht“ wie auch der ,politischen Besetzung des Körpers“ (Foucault 1976, S. 40) verfolgt er das Anliegen, Körper bis in seine Materialität hinein als Effekt von Diskursen und damit von Praktiken der Macht und des Wissen zu bestimmen (Siebenpfeiffer 2008, S. 56). Entsprechend gäbe es „,keine Stelle am und im Körper [...], die nicht diskursiv hervorgebracht wurde" (Siebenpfeiffer 2008, S. 58; vgl. dazu auch Magyar-Haas 2013, S. 141 f.).

Körper erscheint bei Foucault (1976, S. 37) dabei in seiner doppelten Gegebenheit: als unterworfener wie auch produktiver Körper. Der Körper ist zwar unmittelbar im „Feld des Politischen" eingebettet, wo die Machtverhältnisse ihn umkleiden, markieren, dressieren und auch Zeichen von ihm verlangen (vgl. Foucault 1976, S. 37). Zugleich ermöglicht dieser Zugriff auf den Körper - mit einem spezifischen, aus herrschenden Vorstellungen resultierenden Wissen um den Körper - erst seine Hervorbringung: Produziert werde also der Körper durch einen Macht-Wissen-Komplex, zu dem „das Wissen um den Körper mit der Macht über den Körper [verschmilzt]““ (Siebenpfeiffer 2008, S. 56).

Körper als Diskurseffekt zu verstehen heißt indes nicht nur, das „Denken-über“ und „Sprechen-von“ Körpern zu fokussieren. Vielmehr kann - historisch betrachtet - von der Materialisierung von Diskursen im Körper sowie in körperlichen Handlungsvollzügen ausgegangen werden, die bestimmte körperLeibliche Seins-Weisen (etwa Geschlecht, Klasse) hervorbringen. Diese Materialisierung entsteht in und durch performative(n) Akte(n), durch Anrufung bzw. Aufrufung von Regeln (vgl. Duttweiler 2003, S. 32.). Performativität ist dabei als ständig zu wiederholende und zitierende Praxis zu betrachten, durch die der Diskurs die Wirkung erzeugt, die er benennt (vgl. Butler 1997, S. 22). Dass

Verhaltens durch Aufhebung der Verhältnismäßigkeit des Daseins“ fasst (Plessner 1941/2003, S. 366).

9 Es wäre weiter zu analysieren, inwiefern Körper hier als ,Handlungssubjekt ${ }^{6}$ als ,Agens ${ }^{6}$ zu fassen wäre, wie dies sich in der körpersoziologischen Perspektivierung von Gugutzer (2006, 2012) finden lässt. 
regulierende Normen in performativer Wirkungsweise die Materialität der Körper konstituieren, verdeutlicht Judith Butler am prominentesten. KörperLeibliche Seins-Weisen werden zwar kulturell reglementiert, zugleich werden die Regeln durch Re-Präsentationsformen in, auf und durch den Körper re-inszeniert - in welcher Re-Inszenierung gleichwohl auch Verschiebungen und subversive, widerständige Be-Spielungen von Regeln möglich sind. ${ }^{10}$

Dabei ist nicht nur das ,Körperäußere“, sondern auch das ,Körperinnere‘ in ihrer jeweiligen Materialität gesellschaftlichen, diskursiven Formierungs- und Disziplinierungsprozessen ausgesetzt und von Machtverhältnissen überzogen. Dass „Körperselbstbilder die normative Ordnung einer Gesellschaft nicht einfach widerspiegeln [...] sondern dass die empirischen Subjekte das Repertoire der leiblichen Ausdrucksformen in seiner ganzen Breite verwenden, um die ihnen zugemutete normative Ordnung zu bespielen“, legt auch Norbert Axel Richter (2009, S. 31) Foucault kritisch weiterführend dar. Hinsichtlich einer Pointierung des Verhältnisses von Körper und Grenze in Bezugnahme auf poststrukturalistische Debatten und ihrer Weiterführung eröffnet sich (metaphorisch) das Bild von an-grenzenden KörperLeibern. An-grenzend verweist hier auf die spielerische Widerständigkeit machtvollen Diskursen gegenüber. Diese bei Foucault in den frühen Schriften vernachlässigte und von Richter relevant gesetzte Widerständigkeit des KörperLeibes berücksichtigt auch Ulle Jäger, indem sie poststrukturalistische Perspektiven (hinsichtlich des diskursiven, sich materialisierenden Körperwissens) mit phänomenologischen Ansätzen (bezüglich gelebter Erfahrung) zu verknüpfen sucht. So kann nach Jäger (2004, S. 81) der Leib ,sowohl als Ort verstanden werden, an dem die Macht wirksam wird, als auch als potentieller Ort des Widerstandes“"11. Diese Widerständigkeit bietet auch Rückbezüge zu Plessners Konzeptionalisierung des Leibes, der eben keine ,passive Hülle‘ oder etwas Materielles sei, sondern eben ,eine erlebte Grenzfläche gegen die Umwelt“" (Plessner 1941/2003, S. 249). Anders als in der skizzierten anthropologischen Bestimmung des Körpers in seiner Verhältnishaftigkeit zur Umwelt (1.1), rücken in sozialontologischen Lesarten von Judith Butler und Jean-Luc Nancy dezidiert die sozialen, materiellen, ökonomischen Bedingungen und Bedingtheiten des KörperLeibes in den Blick. In diesen Perspektiven wird der KörperLeib in seiner Verletzbarkeit, Verwundbarkeit und Ausgesetztheit - also in seiner Grenzhaftigkeit - betrachtet.

\subsection{Ausgesetzte Körper: Sozialontologische Positionen}

Das ontologische Problem des ,Seins“ sowie das epistemologische Problem der Wahrnehmung und ,framing' von Leben wird bei Butler (2010, S. 9) vor dem Hintergrund der „Operationen der Macht“ diskutiert. In ,Frames of War' plädiert sie für eine „,neue Ontologie des Körpers“, „die mit einem neuen Verständnis von Gefährdung, Schutzlosigkeit,

10 Identität im Sinne einer „Auseinandersetzung mit kulturellen Deutungsmustern und Artefakten, die körperliche Erfahrungen hervorrufen“, werde dann „glaubhaft“, wenn diese Erfahrungen „als Ausdruck des natürlichen Leibes“ gedeutet werden, wenn also körperliche Erfahrung verleiblicht werde (Duttweiler 2003, S. 32; vgl. Magyar-Haas 2013, S. 142).

11 Jäger (2004, S. 81) schlägt vor, den Leib ,dahingehend zu untersuchen, wie an ihm und in ihm die normativen Diskurse wirksam werden“. 
Verletzlichkeit, wechselseitiger Abhängigkeit, Exponiertsein, körperlicher Integrität, Begehren, Arbeit, Sprache und sozialer Zugehörigkeit einhergeht“ (Butler 2010, S. 10). In einem so gefassten Verständnis lässt sich körperLeibliches Sein in seiner Abhängigkeit von Anderen und von gesellschaftlichen, politischen, ökonomischen und ökologischen Bedingungen erkennen. Dabei wäre Gefährdung (,precariousness“"), Exponiertsein, Ausgesetztheit - welche als Charakteristika hier unter dem Begriff der Grenzhaftigkeit subsumiert werden - als eine ,allgemeine Bedingung“ und „nicht einfach als Merkmal dieses oder jenes Lebens zu begreifen“ (Butler 2010, S. 29, herv. i. O.). Mit dieser genuin ontologischen Lesart rücken aber auch die Lebensbedingungen ${ }^{12}$ in den Fokus, die erfüllt werden müssen, damit das Leben lebbar und wahrnehmbar ist (vgl. Butler 2010, S. 29).

Diese Bedingtheit des grenzhaften KörperLeibes von sozialen, materiellen, ökonomischen Bedingungen findet seine Pointierung bei Jean-Luc Nancy (2004, S. 129) in dem Begriff des Mit-Seins. Er konzeptionalisiert „eine Ontologie des Körpers“ - eine Ontologie aller Körper, „unbelebter, belebter, fühlender, sprechender, denkender, wiegender Körper“. Oder anders formuliert: er bestimmt das Sein als „,das singulär plurale Mit-sein“ (Nancy 2004, S. 118). In diesem Ausgesetzt-Sein ${ }^{13}$ dem Draußen gegenüber zeigt sich die ontologische Konstitution des Gemeinsamen. Dabei erweisen sich körperleibliche Wesen als Gleiche, jedoch nicht als miteinander Identische, da sie sich aneinander lediglich ihre jeweilige Andersheit erkennen können (vgl. Nancy 1988, S. 73 f.). Diese Gedanken werden in dem Band ,singulär plural sein“ von 1996 weiterentwickelt, wo Nancy explizit hervorhebt: „,Die Pluralität des Seienden steht am Grund des Seins “ (Nancy 2004, S. 34, herv. i. O.) und entsprechend ,Sein“ - analog zu Butler - auch nicht als ,Zustand“ oder ,Eigenschaft“ fasst, sondern als „Aktion/Passion“, das positioniert und zugleich zu anderen verschiedenen Positionen in Dis-Position (ver)setzt (Nancy 2004, S. 34 f.).

Mit der Formulierung: „Der Körper ist das Sein der Existenz“, legt Nancy in seinem Werk ,Corpus` (2007, S. 18) diese ontologische Perspektive auf Körper explizit dar. Dabei betont er, dass es „das Wesen der Existenz ist, kein Wesen zu haben“, schließlich gehe das Sein dem Körper nicht voraus, vielmehr seien Körper „Existenz-Stätte“, „Stätte der lokalen [changierenden, beweglichen, vielfältigen] Existenz" (Nancy 2007, S. 19, herv. i. O.). So wundert es wenig, dass Nancy Körper nicht im Diskurs oder in der Materie verortet, sondern auf der Grenze, als Grenze: ,äußerer Rand, Bruch und Einschnitt des Fremden in das Kontinuum des Sinns, in das Kontinuum der Materie“ (Nancy 2007, S. 20). Diese Auslegung mag an Plessners (1928/2003, S. 181) bereits skizzierte Bestimmung: „Körper ist die Grenze seiner selbst und des Anderen“ gewissermaßen anschließen. Mithin verweist Nancy (2007, S. 15) darauf, dass in verschiedenen - insbesondere psychoanalytischen Auseinandersetzung versucht wurde, ,den Körper ,bezeichnend“ [significant] zu machen, anstatt die Bedeutung [signification] als das zurückzuweisen, was allenthalben den Raum der Körper entgegensteht“. Als beschriebener Körper, welchen es zu entschreiben gelte

12 Nach Isabell Lorey (2012, S. 34) bezeichnet ,Prekär-Sein“ (precariousness, ,Gefährdung ‘) ,eine vielfältige unsichere Konstituierung von Körpern, die immer sozial bedingt sind“. Es sei - und hier ließe sich auf den Begriff des Leibes ebenfalls verweisen - eine Bedingung, die menschlichem wie auch nicht-menschlichem Leben eigen ist“" (Lorey 2012, S. 34).

13 Anerkennungstheoretisch wäre dabei folgende Erkenntnis anschlussfähig: „daß ich zunächst dem anderen und dem Ausgesetzt-Sein des anderen ausgesetzt bin“ (Nancy 2004, S. 68). 
(vgl. Nancy 2007, S. 15), ist Körper eine Widerspruchsinstanz: es gibt Bedeutung durch und in ihm - als Körperlichkeit der Sprache - oder aber es gebe eine Bedeutung von ihm (vgl. Nancy 2007, S. 61 f.). Sodann verkörpere der bezeichnende Körper ausschließlich „den absoluten Widerspruch, nicht Körper sein zu können ohne das Sein eines Geistes, der ihn entkörpert“ (Nancy 2007, S. 62).

In Anlehnung an Plessner lässt sich Körper als Grenze, an Butler und Nancy in seiner Grenzhaftigkeit bestimmen. Sowohl Grenze als auch Grenzhaftigkeit implizieren dabei eben keinen Rand, keine Um-Randung, bloße Fläche und keinen Strich, vielmehr lassen sie sich als Ausdruck von Zwischen-Verhältnissen, zwischen Innen und Außen (1.1), Selbst und Anderen, zwischen ,singulären Pluralitäten' (1.3) denken. Entsprechend kann der Leib in doppelter Perspektive gefasst werden: zum einen in seiner Grenzhaftigkeit und Ausgesetztheit (1.3), zum anderen in seiner Widerständigkeit, welche ebenfalls auf die Grenzhaftigkeit des Leibes zu verweisen vermag, aber im Sinne von Eigen-Sinnigkeit (1.2). Diese sozialontologischen Perspektivierungen, die KörperLeiber in ihrer Bedingtheit von sozialen, ökonomischen und politischen Bedingung gegenüber verstehen und - in einer anderen Theoriesprache - als ,Produkt' gesellschaftlicher Verhältnisse auslegbar wären, werden im Folgenden erweitert um Lesarten, die KörperLeiber stärker als Produkt und zugleich Produzent des Sozialen - und so auch von Grenzen - auffassen (vgl. Gugutzer 2006).

\subsection{Körperlichkeit sozialer Praktiken: Praxeologische Zugänge}

Die Umgangsweisen mit dem Körper, das Wissen über ihn und das Spüren des Körpers sind von gesellschaftlichen Macht- und Ungleichheitsstrukturen sowie von je spezifischen historischen Gegebenheiten geprägt. Mithin ließe sich Körper mit Pierre Bourdieu (1993) als Produkt historisch sich wandelnder sozialer (Ungleichheits-)Verhältnisse oder mit Michel Foucault (1976) als Produkt von Machttechniken erkennen. ${ }^{14}$ Körper als Produkt sozialer Verhältnisse unterliegt unterschiedlichen Formen der Markierungen und Einschreibungen (vgl. 2). In den Ansätzen von Bourdieu und Erving Goffman wird der Körper darüber hinaus als Produzent des Sozialen betrachtet. Er repräsentiert die sozialen Verhältnisse und agiert in diesen genauso wie er an der Hervorbringung und Aufrechterhaltung dieser mitbeteiligt ist.

Dass sich soziale Verhältnisse sowie ihre Klassifikations- und Deutungsmuster in die Körper von Personen einschreiben und zu habitualisierten Handlungsweisen werden, entfaltet Bourdieu in seinen Ausführungen zu Habitus und Inkorporation. Körper fungiert hier zwar als ,Speicher“ inkorporierter Strukturen und Wissensbestände, zugleich seien auch soziale Ordnungen von der Körperlichkeit sozial handelnder Subjekte beeinflusst (vgl. Bourdieu 1993; Goffman 2003).

14 Die soziale Prägung des Körpers lässt sich insbesondere anhand der in der Frauen- und Geschlechterforschung geführten Debatten um den Geschlechterkörper (vgl. Kessler und McKenna 1987; West und Zimmerman 1987) oder um die durch den Körper legitimierte Geschlechterdifferenz (vgl. Gildemeister 1992) nachzeichnen. Diese finden ihre Pointierung in Judith Butlers (1991) heteronormativitätskritischer Analyse sozialer Geschlechterkonstruktionsprozesse sowie in ihrer Kritik an der Naturalisierung von Geschlecht bzw. von Geschlechterkategorien. 
Die Zugangsweise zum Körper als Produkt und Produzent des Sozialen fügt sich in ein praxeologisches Verständnis sozialer Praktiken ein. Zum einen lässt sich mit Bourdieu herleiten, dass der Habitus als modus operandi fungiert, zum anderen wird in seiner Sozialtheorie ebenso die Körperlichkeit und Materialität von Praktiken hervorgehoben. An diese Deutungen schließt Reckwitz in seiner ,Theorie sozialer Praktiken“ (2003) weiterführend an, indem er sich von einem Handlungs- zugunsten eines Praktikenbegriffs distanziert und entsprechend u. a. die Materialität der Praktiken in ihrer Verankerung in Körpern und Artefakten betont (vgl. Reckwitz 2004, S. 44). Dabei umfasse die Körperlichkeit der Praktiken sowohl die Performativität des Handelns als auch die Inkorporiertheit von Wissen. Praktiken setzen sich aus Körperbewegungen zusammen und bilden ,in der Regel Verhaltensweisen mit Dingen, mit Artefakten [...], in deren Zusammenhang das praktische Wissen aktiviert wird" (Reckwitz 2004, S. 44). Weiterführend kritisiert Reckwitz die Formulierung universeller Aussagen über ,,die“ Routinisiertheit oder ,die “ Unberechenbarkeit" von sozialen Praktiken und plädiert für die Rekonstruktion ,historisch-lokaler' Spezifizität dieser (vgl. Reckwitz 2004, S. 52). Folglich gelte es zu fragen, inwiefern soziale Praktiken hinsichtlich der Re-Produktion ,des' Sozialen oder hinsichtlich ihrer differenten Unberechenbarkeiten ,dem‘ Sozialen gegenüber in den Blick geraten. Ihr subversives Potential und ihre Veränderungsoffenheit werden lediglich beim Letzteren fokussiert.

In diesen körpersoziologischen Perspektivierungen stehen Fragen danach im Mittelpunkt, wie körperLeibliche Praktiken zur Herstellung, Stabilisierung und zum Wandel sozialer Ordnungen beitragen. Somit seien auch Körper an der Konstituierung von Grenzen beteiligt. Ein so gefasstes Verständnis von Grenze weist über die bislang verhandelten Perspektiven auf Körper als Grenze (1.1) bzw. Körper als Grenzhaftes (1.3) hinaus. Indem Körper als Produzent sozialer Verhältnisse betrachtet wird, rückt er selbst als Produzent von Grenzen in den Blick. Daran anschließend lassen sich folgende Fragen formulieren: Inwiefern werden mittels Markierungen am, über oder entlang von Körper(n) Grenzen produziert (2)? Inwiefern werden mit KörperLeibern Grenzen konstituiert (3)? Zur Annäherung an diese Überlegungen wird die analytische Differenzierung zwischen hervorgebrachten symbolischen Grenzen (2) - als Markierungen von und an Körper(n) und sozialen Grenzen (3) - als Praktiken mit Körpern - dimensioniert.

\section{Konstituierung symbolischer Grenzen an und über Körper}

Im folgenden Abschnitt wird diskutiert, welche Bedeutung den Wahrnehmungen, Sichtbarmachungen und (sprachlichen) Markierungen von Körpern in Konstruktionen von Personen und Personengruppen als ,Andere` zukommt. Diese Konstruktionsweisen werden als Konstituierung von symbolischen Grenzen perspektiviert.

Mit Fragen nach KörperLeibern gehen Überlegungen zu dem Verhältnis von Wahrnehmbarkeit; Unsichtbarkeiten und Sichtbarkeiten; von Sagbarem und Unsagbarem; von Repräsentationen und Präsentationen einher, durch welche Grenzen konstituiert werden. Für diese so hervorgebrachten Unterscheidungen sind ,sowohl Zeichen und Bilder von gesellschaftlichen Selbstentwürfen als auch Artikulationen des einzelnen, symbolischen Körpers“" virulent (Sasse und Wenner 2002, S. 11). Um diese Verhältnisse zu entziffern, werden Aspekte der Wahrnehmung, der Dialektik der (Un)Sichtbarkeiten sowie der kör- 
perbezogenen sprachlichen Markierungen näher betrachtet. Dabei interessiert, inwiefern über (hegemoniale) Wahrnehmungsweisen sowie Akte des ,(Nicht-)Sprechens-über ,andere' Körper hervorgebracht und so Grenzziehungen vorgenommen werden.

Die Problematik der Wahrnehmung bzw. Wahrnehmbarkeit lässt sich in Anlehnung an die phänomenologische Ontologie Jean-Paul Sartres (2012) plausibilisieren, die er in sozialen Blickverhältnissen verortet und mit Anerkennungsverhältnissen relationiert. Nach Sartre (2012, S. 467) sei der Akt und Prozess des Wahrnehmens einerseits ein Anblicken und andererseits ,ein Bewußtsein davon [zu] erlangen, angeblickt zu werden“. Unmittelbar erfahrbar für körperleibliche Subjekte wird nicht nur die Anwesenheit von Anderen, sondern auch die Potentialität des Gesehen-Werdens (Sartre 2012, S. 467). Indem bei Sartre das ,Ich“ lediglich und ausschließlich über den bestätigenden, anerkennenden Blick des ,Anderen“ als ,Ich“ erkenn- und erfahrbar sei, wäre in jener Perspektive zum einen das ,Ich“ den Urteilen der Anderen ausgeliefert, zum anderen liegen jedoch zugleich in diesem Blick die Bedingungen der existenziellen Möglichkeiten des ,Ich“ (Sartre 2012, S. 344). Durch Prozesse des ,Angeblickt-Werdens ‘ und die dem Blick zugrunde liegende Verobjektivierung durch die Anderen wird dem körperLeiblichen Sein der Status eines Subjektes und zugleich Objektes zugewiesen. Gleichwohl wird jene Beziehung zwischen dem ,Ich“ und dem (anblickenden) ,Anderen“ bei Sartre als ein Verhältnis unter ,Gleichen' konzipiert (vgl. Sartre 2012, S. 344). Wird also die Frage danach gestellt, inwiefern bestimmte körperleibliche Subjekte ${ }^{15}$ wahrnehmbar und anerkennungswürdig werden, müsse den Formen der Anerkennung das Prinzip der Gleichheit zugrunde gelegt werden. Während Sartre auf das paradoxale Verhältnis der Subjektivierung und Objektivierung in dem Gesehen- und so Erkannt-Werden fokussiert, legt auch Foucault (1976, S. 260) Sichtbarkeit als „eine Falle“ aus - aber in einer anderen Hinsicht ${ }^{16}$. Als Falle ließe sich Sichtbarkeit bezeichnen, da der ,doch so sichtbare Körper gleichsam in seiner Unsichtbarkeit gefangen“" (Foucault 2005, S. 29) zu sein scheint. Hier entfalte sich Körper als Diskurseffekt (1.2) in der Art und Weise, als dass „,derjenige, welcher der Sichtbarkeit unterworfen ist und dies weiß“, die Zwangsmittel der Macht übernehme, diese gegen sich selbst ausspiele - ,er internalisiert das Machtverhältnis, in welchem er gleichzeitig beide Rollen [Sehen und Gesehen-Werden] spielt“ und so „zum Prinzip seiner eigenen Unterwerfung wird“" (Foucault 1976, S. 260).

In Anlehnung an die Darlegung der Dialektik von Subjektivierung und Objektivierung in Prozessen bzw. Akten der Wahrnehmung lässt sich weiter fragen, welche Körper sichtbar, also „wahrnehmbar, erkennbar und anerkennungswürdig werden“ (Butler 2002, S. 168). Entsprechend wäre zum einen zu rekonstruieren, welche Sichtbarkeitsregimes hegemonial werden, zum anderen, inwiefern in der Markierung gewisser körperbezogener Aspekte hegemoniale Sichtbarkeiten entstehen, die ebenfalls symbolische Grenzen konstituieren. Die Wahrnehmbarkeit jener ,Anderen' mag dabei auch der Sicherung symbolischer Grenzziehungen dienen, worin ihre Dilemmata begründet liegen. Indem an und

15 Jean-Paul Sartre legt in ,Das Sein und das Nichts`drei ontologische Dimensionen des Körpers dar: die Existenz des Körpers, die Benutzung und Erkennung des Körpers durch Andere sowie die Verobjektivierung (Sartre 2012, S. 619).

16 Zur weiterführenden Analyse von Sichtbarkeiten bei Sartre und Foucault vgl. Neckel 1991, S. $25 \mathrm{ff}$. 
über Körper Markierungen, Zuschreibungen und Unterscheidungen vorgenommen werden, ließe er sich mithin als Austragungsort von Sicht- und Sagbarkeitsregimen deuten. Oder mit Yuval-Davis (2006, S. 199) argumentiert: kategoriale Attributierungen konstruieren Grenzen, ,that differentiate between self and other, determining what is ,normal and what is not, who is entitled to certain resources and who is not. In this way the interlinking grids of differential positionings in terms of class, race and ethnicity, gender and sexuality, ability, stage in the life cycle and other social divisions, tend to create, in specific historical situations, hierarchies of differential access to a variety of ressources economic, political and cultural".

In dem ,Sprechen-über‘ werden körperbezogene Aspekte wie Geschlecht, Ethnie, Alter, Gesundheit relevant gesetzt und dadurch , andere', konstruierten Normen bzw. normativen Erwartungen (partiell) nicht entsprechende Körper hervorgebracht. So werden also über Wahrnehmungs- und Deutungsweisen, über Sicht- und Sagbarkeiten sowie über und an Körper(n) Grenzen des ,Normalen“ wie auch des ,Nicht-Normalen', des ganz ,Eigenen` und des ,Anderen` konstruiert, bestätigt, verschoben und aufgelöst (vgl. Burghard 2014). Dabei werden bereits bestehende Wahrnehmungsmuster ,anderer ' Körper re-produziert (vgl. Nieke 1993). Über die Anrufung körperbezogener Aspekte werden Personen zu: ,Männern', ,Frauen“, ,Homo-‘ und ,Heterosexuellen“, ,Migrant_innen“, ,Alten', ,Behinderten', ,Dicken', ,Dünnen“ - kurzum zu ,Anderen' - gemacht und entsprechend auch zu Adressatinnen und Adressaten Sozialer Arbeit (vgl. Mörgen 2014; Burghard 2014). Denn es sind bestimmte - ,andere“: ,unansehnliche', ,ungesunde“, ,faule‘, ,überarbeitete - Körper, die in gesellschaftlich konstruierte sowie symbolisch konnotierte Aufregungszonen fallen und zum Anlass sozialer Ausschließung oder aber sozialpädagogischer Unterstützungsleistungen werden.

Exemplarisch lässt sich am medial inszenierten ,Unterschichtskörper', der das ,ganz andere' einer Mehrheitsgesellschaft zu repräsentieren scheint, nachzeichnen, dass sich das jeweils Andere, das ,Nicht-Wir' am Eigenen, dem ,Wir', in der Art manifestiere, dass sich biologisierende und kulturalisierende Vorstellungen mit Annahmen über körperliche Handlungsweisen und -vollzüge verknüpfen, welche bspw. als Begründung für ethnische, nationale aber auch klassenspezifische Zugehörigkeiten hinzugezogen werden. Die diskursive Hervorhebung eines als prekär markierten Körpers diene dazu, das Bild eines von Armut betroffenen Körpers sichtbar zu repräsentieren, zu moralisieren und zu kulturalisieren. Adressiert wird jedoch der subjektive, ganz eigene Umgang mit dem Körper und die ihm eingelagerten Handlungsweisen, welche es sozialpädagogisch zu bearbeiten ${ }^{17}$ gelte (vgl. Kessl 2013, S. 128 ff.).

Gerade bei minorisierten Gruppen können jene Formen des Angeblickt-Werdens als auch des (Un)Sichtbar-Gemacht-Werdens als gewaltvolle Akte betrachtet werden, welche

$17 \mathrm{Zu}$ fokussieren wäre dabei, welchen normierenden Praktiken der als prekär markierte Körper unterworfen wird, um dadurch gesellschaftliche Normierungs- und Normalisierungsprozesse zu legitimieren. Weiterführend, in ungleichheits- und differenztheoretischer Perspektive, ließe sich als Frage formulieren, inwiefern sich unterschiedlich (sozial, materiell, kulturell) hervorgebrachte Differenzen als körperliche Differenzen einerseits materialisieren und andererseits als diskursiv hervorgebrachte Konstruktionen von Körperlichkeit sowohl als Grundlage als auch als Legitimation für soziale Ungleichheit - sprich als „verKörperte Ungleichheit“ (Mörgen 2014) - herangezogen werden (vgl. Schmincke 2009). 
symbolische Grenzziehungen an und über Körper konstituieren. In einer solchen Konzeptionalisierung von Blickverhältnissen - wie auch im Rückgriff auf Sartre dargelegt wurde - ist die leibliche Dimension insofern angelegt, als der Körper nicht nur als Manifestation gesellschaftlicher Macht- und Ordnungsverhältnisse, sondern auch als ,Ort der Erfahrung des Selbst' in den Blick zu nehmen sei (vgl. Mörgen 2014).

Ausgehend davon, dass über Formen und Weisen der Versprachlichung wie auch des Sehens und Gesehen-Werdens bestimmter körperLeiblicher Seins-Weisen Grenzen produziert werden, erscheint jedoch insbesondere die Sprache körperlos, „das was gesagt wird, ist körperlos, ist das ganze Unkörperliche der Welt", und zugleich ist der Akt des Sagens körperlich, ,als hörbare Stimme oder sichtbarer Strich“ (Nancy 2004, S. 130) ${ }^{18}$. Konstituierungen von Grenzen vollziehen sich an und über Körper(n), sowie auch überdies hinaus mit Körpern. Letzteres wird im Folgenden mit kritischem Bezug auf die Hervorbringung von Gemeinschaften und weiteren sozialen Formationen diskutiert.

\section{Konstituierung sozialer Grenzen mit Körpern}

Wurde im vorausgehenden Kapitel dargelegt, wie durch die Relevantsetzung körperbezogener Aspekte hegemoniale Bestimmungen vorgenommen und Grenzen zwischen dem gezogen werden, was als ,normal' und als ,abweichend' gilt, wird in den folgenden Überlegungen die Bedeutung des KörperLeibes für das Soziale (vgl. 1.4) diskutiert. Um dies zu verdeutlichen, wird auf die Konstituierung von Kollektivität und ,Gemeinschaft ‘ als körperLeibliche Praktik eingegangen. Aufgezeigt werden soll dabei, inwiefern in der körperLeiblichen Konstituierung sozialer Formationen bereits ein ,Außen“ produziert wird und in dieser Produktion Grenzen gezogen werden. Im Vordergrund steht dabei weniger die Hervorbringung von Gemeinschaften, als vielmehr die Frage nach ihrer grenzkonstituierenden Bedeutung.

Auf die Relevanz von Körperpraktiken bei der Hervorbringung des pädagogischen Alltags gehen verschiedene ethnographische Studien (vgl. Kuhn 2013; Schulz 2010) auf erziehungswissenschaftlich reformulierte performativitätstheoretische (vgl. Wulf et al. 2001) und/oder praxeologische (vgl. Reckwitz 2003) Ansätze Bezug nehmend - ein. In diesen Arbeiten wird der Begriff des Performativen in der sprachphilosophischen Auslegung Austins insofern modifiziert, als sie den Fokus auf die körpersprachliche Dimension der Wirklichkeitskonstruktion setzen, also auf soziale Praktiken, die körperlich sind (vgl. Magyar-Haas 2014, S. 23). Mit der Hinwendung zum Performativen - auch im Kontext der Sozialen Arbeit - rücken Interaktionen, der Inszenierungs- und Aufführungscharakter sozialen Handelns, dramaturgische Sprech- und Handlungsvollzüge, sowie die Körperlichkeit und Materialität von Erziehungs- und Bildungssituationen in den Mittelpunkt (vgl. Wulf 2005). Die Bestimmung des Körpers als Produkt wird um die Perspektive auf Körper als Produzent des Sozialen ergänzt (1.4). Entsprechend wird die Frage nach der Beteiligung der KörperLeiber an der Re-Produktion von sozialen Ordnungen und so von Grenzziehungen gestellt.

18 „Das Körperlose exponiert die Körper gemäß ihres Mit-ein-ander-sein: nicht isoliert, nicht vermischt [confondu], sondern unter sich als Ursprünge“ (Nancy 2004, S. 130, herv. i. O.). 
Inszenierungen von und mit Körpern vermögen Gemeinschaften und weitere - als geschlossen erscheinende - soziale Formationen konstituieren. Dabei kommt etwa sozialen Ritualen - im Sinne von Formen der Praxis, die diese zugleich hervorbringen - aufgrund ihres performativen Charakters eine verstärkende Bedeutung zu (Wulf et al. 2001, S. 115 ff.). Auf ritualtheoretische Perspektiven Bezug nehmend untersuchen MagyarHaas und Kuhn (2011) die Konstituierung von Gruppen und Machtverhältnissen in Kreissituationen im Kontext der Frühen Kindheit und Offener Jugendarbeit. Sie differenzieren dabei zwischen einer räumlich-materiellen und symbolisch-ideellen Dimension der Hervorbringung von Gruppen und Zugehörigkeiten. Während erstere die leibliche Anwesenheit der Teilnehmer_innen und die (regel)konforme Aufführung des Kreisrituals (der Platzierung der Körper im Kreis) voraussetzen, wird der symbolische Kreis mittels Anrufung von Normen und kollektiv zu teilenden Regeln konstruiert. Diese beiden Ebenen sind keinesfalls deckungsgleich, sodass eine leibliche ,Kopräsenz' noch keine legitime Mitgliedschaft zu einer ideellen, Gemeinschaft' garantiert. Gerade bei der Jugendeinrichtung wurden regelmäßige Teilnahme an dem sozialpädagogischen Projekt sowie Leistungsorientierung als zentrale Kriterien der Zugehörigkeit relevant gesetzt - wobei die so markierten Grenzen der Zugehörigkeit seitens der Jugendlichen subtil oder offensiv hinterfragt wurden (vgl. Magyar-Haas und Kuhn 2011, S. 25 f., 30). Während die Anordnung der Körper im Kreis, die potentielle gegenseitige Beobachtbarkeit bei der Jugendgruppe bereits disziplinierend schien, erwies sich im Kindergarten der Zugriff auf die Körper der aus dem Kreis schwinden wollenden Kinder als legitim, um die ,Gemeinschaft‘ zusammen zu halten (vgl. Magyar-Haas und Kuhn 2011, S. 29 f.). In diesen Bestimmungen und körperLeiblichen Inszenierungen von Ritualen entstehen ,Kollektivkörper ${ }^{\text {- mit }}$ welchem Begriff Sasse und Wenner (2002, S. 11) „die Beziehungen der Teile im bzw. als Körper und zwischen den ,Körpern“" akzentuieren, in Abgrenzung zu Termini wie ,Masse“ oder ,Meute': „Es scheint im Kollektivkörper verdoppelt, was die Frage nach dem Körper selbst schon offen lässt: das Ineinander von gemeinschaftlich imaginärer und symbolischer Figurierung und körperlicher Teilhabe im Konflikt von Abgrenzung, Verbindung, Regulierung und Exotopie“" (Sasse und Wenner 2002, S. 11). Entsprechend werden mit Körpern in ,Gemeinschaften“ oder in geschlossenen Gruppen einem ,Außen“ gegenüber Grenzen gezogen und somit ein ,Innen' konstruiert.

Wurde im Kapitel zwei die Hervorbringung symbolischer Grenzen mittels Markierungen bestimmter körperbezogener Aspekte dargelegt, so wurden im dritten Teil die mit Körpern hervorgebrachten Formationen samt ihres - soziale Grenzen konstituierenden Charakters berücksichtigt. Dass diese beiden Dimensionen Ausdruck einer analytischen Trennung sind, lässt sich etwa anhand des Begriffes der ,Leistungsgesellschaft ‘ illustrieren: Semantisch ist an diesem Terminus erkennbar, wer zu dieser symbolisch dazugehört (die ,Leistungswilligen') und wer nicht (die ,Nicht-Leistungswilligen'). Konstruiert wird dabei - einerseits die Dimension der Macht in den gemeinschaftlichen Bezügen genauso vernachlässigend wie andererseits Fixierungen, Festschreibungen auf bestimmte Merkmale propagierend - ein Mythos der Homogenität ,der Leistungswilligen“. Aber auch die explizite oder implizite Hervorhebung bestimmter (körperbezogener) Aspekte, wie etwa Leistungsfähigkeit, Disziplin oder Fleiß (vgl. Heite et al. 2013), simuliert ein ,Wir', das sich vordergründig durch Ähnlichkeiten, gemeinsame Züge, Gleichrangigkeit nach Innen auszeichnet. Darin vermag es ein ,Nicht-Wir' zu produzieren und nach Außen hin (dyna- 
mische) Grenzen zu setzen und diese zu markieren. Derartige Vorstellungen homogener Gemeinschaften als mythische Konstrukte zu entlarven, ist das Anliegen dekonstruktivistischer Denker_innen (vgl. Nancy 1988).

In Anlehnung an und Weiterführung diese(r) Perspektiven ließen sich Körperpraktiken nicht nur als bloß affirmativ im Sinne ihrer Hervorbringungen und Re-Produktion der jeweiligen sozialen Ordnungen sowie Grenzen produzierenden Gemeinschaften betrachten, sondern diese immer auch als kreativ, subversiv und eigensinnig bespielend und unterlaufend (vgl. 1.2.). Sozial relevant ist dieses eigensinnige Handeln deswegen, weil ihm gerade auch widerständiges Potenzial innewohnt (vgl. Gugutzer 2012, S. 54 ff.). Mit der Berücksichtigung dieser Ebene körperlichen Handelns können körperLeibliche, mitunter widerspenstige, Verhaltensweisen in den Blick genommen werden, die ebenso daran beteiligt sind, die Re-Produktion sozialer Ordnungen und Gemeinschaften zu irritieren. So gesehen erweist sich der selbst Grenzen produzierende KörperLeib in seiner Widerständigkeit gegenüber den Grenzen als Grenze.

\section{Ausblickendes Resümee}

Die Problematik des Begriffs der Grenze zeigt sich auf zwei Ebenen: zum einen wird der Terminus mittels seiner konjunkturellen Verwendung sowie seines symbolischen und metaphorischen Charakters - wie der letzte Satz des Kap. 3 dies zu veranschaulichen mag - verschleiert und entgrenzt. Zum anderen erfährt der Begriff eine Engführung, wenn er in wissenschaftlichen Auseinandersetzungen überwiegend mit Nationalstaat(lichkeit) und so in rein raumbezogenen Lesarten verwendet wird. Um diesen Problematiken der Entgrenzung und Engführung der Grenze systematisch und analytisch zu begegnen, wurden Begriffe von Grenzen in Bezugnahme auf die körperLeibliche Dimensionen ihrer Konstituierung hin konturiert.

Im ersten Teil wurde das Verhältnis von Körper, Leib und Grenze auf Basis theoretischer Auseinandersetzungen in ihrer Vielfalt rekonstruiert. So ließ sich mit der anthropologischen Perspektive Helmuth Plessners Körper als Grenze bestimmen. Grenze impliziert dabei weniger etwas Starres, Unwandelbares, vielmehr lässt sie sich als provisorisches Resultat interaktiver Prozesse zwischen KörperLeib und Umwelt denken (1.1). In Anlehnung an poststrukturalistische Perspektiven wurde Körper als Materialisierung von machtvollen Diskursen erörtert. Dass Körper sich im Zuge performativer Äußerungen diesen Diskursen gegenüber als widerständig, sozusagen an-grenzend, erweisen mögen wurde mit Butlers Weiterführung von Foucault entfaltet (1.2). In Bezugnahme auf sozialontologische Lesarten wurde dann der KörperLeib in seiner Ausgesetztheit und Grenzhaftigkeit dargelegt. Mit dieser Auslegung geraten über eine rein subjektbezogene Perspektive hinaus auch soziale, politische und ökonomische Bedingungen und Bedingtheiten des grenzhaften KörperLeibes in den Blick, sowie die Möglichkeiten seiner Wahrnehmung, die über eine normative ,Rasterung' hinausweisen können (1.3). Mittels praxeologischer Zugänge wurde der Körper als Produkt und Produzent des Sozialen perspektiviert. An und über ihn werden genauso Grenzen hervorgebracht wie er selbst an der Konstituierung von Grenzen beteiligt ist (1.4). 
In dem zweiten Teil wurde dargelegt, inwiefern über unterschiedliche Formen und Weisen der Markierung von und an Körpern, durch Wahrnehmung, Relevantsetzung, Deutung sowie Sprechen-Über körperbezogene Aspekte symbolische (und sich materialisierende) Grenzen hervorgebracht werden. Damit werden auch Bereiche des ,Normalen“ und des ,Abweichenden“ des Anerkennungsfähigen und Nicht-Anerkennungsfähigen, des Sichtbaren und Nicht-Sichtbaren abgesteckt (vgl. Heite et al. 2013), Personen in diese positioniert und entsprechend von Sozialer Arbeit adressiert. Diese Grenzziehungen qua Normen legen vorgängig fest, „welches Leben lebenswert, bewahrenswert“ aber auch ,betrauerbar' sei (Butler 2010, S. 57). Sozialwissenschaftlich relevant sind solche Analysen von Grenzkonstituierungen insbesondere dann, wenn körperbezogene Attributierungen die Möglichkeiten des Zugangs zu sozialer Teilhabe, sozial relevanten Ressourcen oder Formen der Anerkennung in spezifischer Weise präformieren (2).

Im dritten Part wurde danach gefragt, wie mit Körpern in konkreten sozialen Praktiken soziale Grenzen produziert werden: etwa zwischen Selbst und Anderen, Individualund Kollektiv-Körpern (vgl. Sasse und Wenner 2002), zwischen ,Wir ‘ und ,Nicht-Wir ‘, Zugehörigkeiten und Nicht-Zugehörigkeiten. Unter dem Begriff der ,sozialen Grenze“ wurden von Akteur_innen in sozialen Situationen mit ihren KörperLeibern hervorgebrachte Grenzziehungen gefasst, also nicht die Materialisierung von symbolischen Grenzen, wie etwa Lamont und Molnár dies entwerfen (vgl. Heite et al. 2013; Heite 2013). Dabei kommt auch in den Blick, inwiefern kollektiv ,geordnete' KörperLeiber an der Re-Produktion sozialer Ordnung beteiligt sind und inwiefern sie - durch die Konstituierung einer Grenze zwischen einem ,Innen` und ,Außen` - exkludierend sein mögen (3).

Entsprechend der hier vorgenommenen Systematisierungsvorschlägen über die Verhältnisse von KörperLeibern und Grenzen ließe sich als Aufgabe erziehungswissenschaftlicher und sozialpädagogischer Forschung formulieren, zusätzlich zu den historisch, sozial und kulturell divergenten Bedingungen der Produktion von Grenzkonstituierungen und Grenzverhandlungen auch die körperLeiblichen Dimensionen explizit zu berücksichtigen. Es gelte also danach zu fragen, welche Normen die Wahrnehmung der KörperLeiber ,rahmen', inwiefern durch die Markierungen körperbezogener Aspekte Grenzen zwischen Anerkennbarkeit und Nicht-Anerkennbarkeit gezogen werden und inwiefern bestimmte körperLeibliche Formen des Sozialen bereits Grenzen zu setzen vermögen. Wird Soziale Arbeit als Grenzbearbeiterin bestimmt, wäre auch zu berücksichtigen, inwiefern sie dabei selbst an der Ko-Produktion von Grenzen beteiligt ist.

Diese hier subsumierend skizzierten Prozesse und Praktiken der körperLeiblichen Konstituierungen von Grenzen empirisch zu re-konstruieren und theoretisch zu de-konstruieren wäre eine not-wendige Aufgabe einer kritischen, (selbst)reflexiven Sozialpädagogik.

Open Access: Dieser Artikel unterliegt den Bedingungen der Creative Commons Attribution Noncommercial License. Dadurch sind die nichtkommerzielle Nutzung, Verteilung und Reproduktion erlaubt, sofern der/die Originalautor/en und die Quelle angegeben sind. 


\section{Literatur}

Bourdieu, P. (1993). Sozialer Sinn. Kritik der theoretischen Vernunft. Frankfurt a. M.: Suhrkamp.

Burghard, A. B. (2014). Körperlichkeit und Leiblichkeit. In K. Böllert (Hrsg.), Kompendium Kinder- und Jugendhilfe. Wiesbaden: SpringerVS (i. E.).

Butler, J. (1991). Das Unbehagen der Geschlechter. Frankfurt a. M.: Suhrkamp.

Butler, J. (1997). Körper von Gewicht. Frankfurt a. M.: Suhrkamp.

Butler, J. (2002). Body: recognizable/unrecognizable. Über das Stück Körper von Sasha Waltz. In S. Sasse \& S. Wenner (Hrsg.), Kollektivkörper. Kunst und Politik von Verbindung (S. 165168). Bielefeld: transcript.

Butler, J. (2010). Raster des Krieges. Warum wir nicht jedes Leid beklagen. Frankfurt a. M.: Campus.

Duttweiler, S. (2003). Body-Consciousness - Fitness - Wellness - Körpertechnologien als Technologien des Selbst. Widersprüche: Selbsttechnologien - Technologien des Selbst, 23(87), 31-44.

Fischer, J. (2000). Exzentrische Positionalität. Plessners Grundkategorie der Philosophischen Anthropologie. Deutsche Zeitschrift für Philosophie, 48(2), 265-288.

Foucault, M. (1976). Überwachen und Strafen. Die Geburt des Gefängnisses. Frankfurt a. M.: Suhrkamp.

Foucault, M. (2005). Die Heterotopien. Der utopische Körper. Frankfurt a. M.: Suhrkamp.

Gildemeister, R. (1992). Die soziale Konstruktion von Zweigeschlechtlichkeit. In I. Ostner \& K. Lichtblau (Hrsg.), Feministische Vernunftkritik. Ansätze und Traditionen (S. 220-239). Frankfurt a. M.: Campus.

Goffman, E. (2003). Wir alle spielen Theater. Die Selbstdarstellung im Alltag. München: Piper.

Gugutzer, R. (2006). Body turn. Perspektiven der Soziologie des Körpers und des Sports. Bielefeld: transcript.

Gugutzer, R. (2012). Verkörperungen des Sozialen. Neophänomenologische Grundlagen und soziologische Analysen. Bielefeld: transcript.

Heite, C. (2013). Setting and crossing boundaries: Professionalization of social work and social work professionalism. In C. Heite, F. Kessl, \& S. Maurer (Hrsg.), Social Work \& Society - special issue social work as working at the border. Verfügbar unter: http://www.socwork.net/sws/ article/view/334. Zugegriffen: 14. März 2014.

Heite, C., Pomey, M., \& Spellenberg, C. (2013). Ein- und Ausschließungspraktiken als Konstituierung von Grenzen. Soziale Passagen. Journal für Empirie und Theorie Sozialer Arbeit, 5(2), 245-257.

Jäger, U. (2004). Der Leib, der Körper und die Soziologie. Entwurf einer Theorie der Inkorporierung. Königstein i. Taunus: Ulrike Helmer.

Kessl, F. (2009). Soziale Arbeit als Grenzbearbeiterin. Einige grenzanalytische Vergewisserungen. In S. Neumann \& P. Sandermann (Hrsg.), Kultur und Bildung. Neue Fluchtpunkte für die Forschung? (S. 43-61). Wiesbaden: VS Verlag für Sozialwissenschaften.

Kessl, F. (2013). Soziale Arbeit in der Transformation des Sozialen. Eine Ortsbestimmung. Wiesbaden: SpringerVS.

Kessl, F., \& Maurer, S. (2010). Praktiken der Differenzierung als Praktiken der Grenzbearbeitung. Überlegungen zur Bestimmung Sozialer Arbeit als Grenzbearbeiterin. In F. Kessl \& M. Plößer (Hrsg.), Differenzierung, Normalisierung, Andersheit. Soziale Arbeit als Arbeit mit Anderen (S. 154-169). Wiesbaden: VS Verlag für Sozialwissenschaften.

Kessler, S., \& McKenna, W. (1987). Gender. An Ethnomethodological Approach. Chicago: The University of Chicago Press.

Kuhn, M. (2013). Professionalität im Kindergarten. Eine ethnographische Studie zur Elementarpädagogik in der Migrationsgesellschaft. Wiesbaden: SpringerVS.

Lorey, I. (2012). Die Regierung des Prekären. Berlin: Turia + Kant. 
Magyar-Haas, V. (2012). Ambivalent concepts of the border: Political borders - bodily boundaries. Social Work \& Society. International Online Journal, 10(2), 1-13. Verfügbar unter: http:// www.socwork.net/sws/article/view/336. Zugegriffen: 14. März 2014.

Magyar-Haas, V. (2013). Körper. In S. Andresen, C. Hunner-Kreisel, \& S. Fries (Hrsg.), Erziehung - ein interdisziplinäres Handbuch (S. 137-146). Stuttgart: J. B. Metzler.

Magyar-Haas, V. (2014). Körperleib-Verhältnisse in Räumen Offener Jugendarbeit. In R. Gräfe \& M. Witte (Hrsg.), Körper und Bewegung in der Sozialen Arbeit. Schwerpunktheft Sozialmagazin, 39(1-2), 22-29.

Magyar-Haas, V., \& Kuhn, M. (2011). Die Quadratur des Kreises? Formierungen von Gruppen und Machtverhältnissen in pädagogisch arrangierten Kreissituationen. neue praxis, 41(1), 19-34.

Mörgen, R. (2014). verKörperte Ungleichheiten und Soziale Arbeit. In N. Langsdorff (Hrsg.), Intersektionalität und Jugendhilfe. Leverkusen: Barbara Budrich, (i. E.).

Nancy, J. L. (1988). Die undarstellbare Gemeinschaft. Stuttgart: Schwarz.

Nancy, J. L. (1996/2004). singulär plural sein. Zürich: diaphanes.

Nancy, J. L. (2007). corpus. Zürich: diaphanes.

Neckel, S. (1991). Status und Scham. Zur symbolischen Reproduktion sozialer Ungleichheit. Frankfurt a. M.: Campus.

Nieke, W. (1993). Chancengleichheit oder Leistungsprinzip. BuE, 46(3), 329-344.

Plessner, H. (1928/2003). Die Stufen des Organischen und der Mensch. Einleitung in die philosophische Anthropologie. In G. Dux, O. Marquard \& Elisabeth Ströker (Hrsg.), Helmuth Plessner: Gesammelte Schriften IV. Frankfurt a. M.: Suhrkamp.

Plessner, H. (1941/2003). Lachen und Weinen. Eine Untersuchung der Grenzen menschlichen Verhaltens. In G. Dux, O. Marquard \& Elisabeth Ströker (Hrsg.), Helmuth Plessner: Gesammelte Schriften VII (S. 201-387). Frankfurt a. M.: Suhrkamp.

Reckwitz, A. (2003). Grundelemente einer Theorie sozialer Praktiken. Eine sozialtheoretische Perspektive. ZfS, 32(4), 282-301.

Reckwitz, A. (2004). Die Reproduktion und Subversion sozialer Praktiken. Zugleich ein Kommentar zu Pierre Bourdieu und Judith Butler. In K. H. Hörning \& J. Reuter (Hrsg.), Doing Culture. Neue Positionen zum Verhältnis von Kultur und sozialer Praxis (S. 40-54). Bielefeld: transcript.

Richter, N. A. (2009). Die Machtverhältnisse überziehen das Körperäußere. Uniformierte Körper im Nationalsozialismus und im Liberalismus. Journal für politische Bildung, 2, 28-36.

Sartre, J. P. (2012). Das Sein und das Nichts. Versuch einer phänomenologischen Ontologie. Hamburg: RoRoRo.

Sasse, S., \& Wenner, S. (Hrsg.). (2002). Kollektivkörper. Kunst und Politik von Verbindung. Bielefeld: transcript.

Schmincke, I. (2009). Gefährliche Körper an gefährlichen Orten. Eine Studie zum Verhältnis von Körper, Raum und Marginalisierung. Bielefeld: transcript.

Schulz, M. (2010). Performances: Jugendliche Bildungsbewegungen im pädagogischen Kontext. Eine ethnographische Studie. Wiesbaden: VS Verlag für Sozialwissenschaften.

Siebenpfeiffer, H. (2008). Der Körper als Diskurseffekt. Michel Foucaults Philosophie der Körperlichkeit. Der Blaue Reiter. Journal für Philosophie: „Unser Körper. Zwischen Ich und Welt“, 26(2), 56-60.

West, C., \& Zimmermann, D. (1987). Doing gender. Gender \& Society, 1, 125-151.

Wulf, C., Althans, B., Audehm, K., Bausch, C., Göhlich, M., Sting, S., Tervooren, A., WagnerWilli, M., \& Zirfas, J. (2001). Das Soziale als Ritual. Zur performativen Bildung von Gemeinschaften. Opladen: Leske + Budrich.

Wulf, C. (2005). Zur Genese des Sozialen. Mimesis, Performativität, Ritual. Bielefeld: transcript.

Yuval-Davis, N. (2006). Intersectionality and Feminist Politics. European Journal of Women's Studies, 13(3), 193-209. 